\title{
Making Sense of Algorithms in Discrete Mathematics
}

\author{
Timothy H. Lehmann ${ }^{1}$ (D) \\ Received: 21 December 2020 / Accepted: 13 April 2021 / Published online: 13 May 2021 \\ (C) Ministry of Science and Technology, Taiwan 2021
}

\begin{abstract}
Network analysis is a topic in secondary mathematics education of growing importance because it offers students an opportunity to understand how to model and solve many authentic technology and engineering problems. However, very little is known about how students make sense of the algorithms typically used in network analysis. In this study, I used the Hungarian algorithm to explore how students make sense of a network algorithm and how it can be used to solve assignment problems. I report the results of a design-based research project in which eight Year 12 students participated in a teaching experiment that spanned four 60-min lessons. A hypothetical learning trajectory was developed in which students were introduced to the steps of the Hungarian algorithm incrementally. The results suggest that students made sense of the intermediate steps of the algorithm, the results of those steps, and how the algorithm works to solve assignment problems. The difficulties that students encountered are also discussed.
\end{abstract}

Keywords Discrete mathematics $\cdot$ Algorithms $\cdot$ Hungarian algorithm $\cdot$ Sense making

\section{Introduction}

Network analysis is a topic of increasing importance in high school mathematics education because it offers students an opportunity to learn how to model and solve problems that they are likely to encounter in their future working lives, particularly in occupations involving technology and engineering (DeBellis \& Rosenstein, 2004; Hart, 2008; National Research Council, 2013). Assignment problems are one such class of problems that can be solved using network analysis techniques, which students might encounter in occupations involving operations and project management. For example, the problem of assigning workers to a set of tasks, so that the total time taken to

Timothy H. Lehmann

t2.lehmann@qut.edu.au

1 School of Teacher Education and Leadership, Faculty of Creative Industries, Education and Social Justice, Queensland University of Technology, E Block, Level 3, Victoria P ark Road, Kelvin Grove, Queensland 4059, Australia 
complete the tasks is minimized, can be modelled using bipartite graphs and solved using the Hungarian algorithm (Kuhn, 1955). Researchers argue that discrete mathematics topics such as network analysis provide opportunities for students to develop algorithmic thinking, which is a foundational aspect of computer science (Gaio \& Di Paola, 2018; Gibson, 2012; Goldin, 2010). Indeed, learning to develop and use algorithms is a key practice in computational thinking, which offers students opportunities to connect mathematics with other STEM disciplines (Pérez, 2018).

The importance of network analysis topics is underscored by their inclusion high school mathematic curriculum around the world (Hart \& Martin, 2018; Ouvrier-Buffet, 2020). In Australia, Year 12 students are introduced to graphs and networks in the General Mathematics curriculum (Australian Curriculum Assessment and Reporting Authority, 2020), and are required to learn a number of algorithms to solve network problems such minimal connector problems (Prim's algorithm), network flow problems (maximum-flow minimum-cut theorem), and assignment problems (Hungarian algorithm). However, very little is known about how students make sense of these algorithms and their competence in using them to solve network problems (Ferrarello \& Mammana, 2018; Hart \& Martin, 2018). Moreover, the prerequisite knowledge required to understand how the algorithms were developed, or their proofs, is beyond the scope of the General Mathematics course. This presents a significant instructional dilemma in that students are required to execute the algorithms without necessarily understanding how or why they work. The purpose of this study is to confront this dilemma by exploring an instructional intervention designed to enhance students' understanding of network algorithms. The Hungarian algorithm is the focus of this study; however, I envisaged that the features of the instructional intervention would be applicable to the teaching and learning of other network algorithms.

\section{Assignment Problems}

An assignment problem is a combinatorial optimization problem that arises when there are a number of tasks $(n)$ and a number of agents available $(n)$ to complete those tasks, each of whom differs in the efficiency with which they can complete each task. If a cost (expressed as a positive, rational number) is assigned to each agent for each task, then an optimum assignment is achieved when one agent is assigned to each task such that the total cost of the assignment is minimized (Kuhn, 1955; Munkres, 1957). For any assignment problem, there are $n$ ! possible combinations, several of which may be optimal (Munkres, 1957). Problems involving a $2 \times 2$ or $3 \times 3$ matrix typically can be solved by inspection, since they contain only 2 and 6 combinations, respectively. However, problems involving larger matrices contain many more combinations, which demand a more efficient method of determining the optimum allocation.

In 1955, Harold Kuhn developed and published an algorithm that can be used to determine optimum solutions to assignment problems. He named the algorithm the "Hungarian method" because it was based on earlier work by two Hungarian mathematicians, Dénes Kőnig and Jenő Egerváry (Kuhn, 1955). In 1957, James Munkres reviewed the algorithm (Munkres, 1957), and it has since been called the KuhnMunkres algorithm or the Hungarian algorithm. The Hungarian algorithm contains three procedures that reduce the value of the cost for each agent to perform each task, 
revealing the comparative costs of alternative options for an optimum assignment. Appendix contains the steps of the algorithm in the form used in the present study.

\section{Review of Related Literature}

Research into the teaching and learning of graph theory and network algorithms is relatively limited, which probably reflects the fact that the field of discrete mathematics itself is relatively new (Ouvrier-Buffet, 2020). In the context of an undergraduate computer science course, Dagdilelis and Satratzemi (1998) identified two broad categories that characterize students' difficulties in understanding graph algorithms, such as the Hungarian algorithm, Dijkstra's algorithm, and Kruskal's algorithm. First, students have difficulty making sense of the details of an algorithm, even when they understand how the algorithm works in general. For example, a student might understand that the Hungarian algorithm narrows the options available for an optimum assignment but have difficulty making sense of the specific steps, such as the reason for covering each row and/or column that contains a zero at the end of each step. Second, students experience difficulty in making sense of the intermediate steps of an algorithm and the results of these intermediate steps. For example, a student may be able to complete the row reduction step but not make sense of the residuals resulting from the reduction procedure.

In response to these difficulties, Dagdilelis and Satratzemi (1998) suggest that educational software can be used to help students visualize the steps of Dijkstra's algorithm and Kruskal's algorithm. More recently, Makohon, Cetin, Nguyen, and Ng (2016) used a similar approach in developing a Java computer animation for the Hungarian algorithm, which displays each step of the algorithm on the screen with a voice commentary. A similar tool is available online at hungarianalgorithm.com. Research into how students use such tools and make sense of the explanations provided at each step appears to be limited. Moreover, these instructional approaches are intended to be used by undergraduate STEM students. More research into instructional approaches suitable for senior mathematics students is needed.

Hart and Martin (2018) argue that discrete mathematics problems offer students an opportunity to model real-world problems and engage in algorithmic problem-solving to develop solutions to those problems. Hart (1998) has previously proposed an instructional pattern that can be used to develop students' algorithmic problemsolving competence. First, the teacher poses a problem, and the students develop a model of that problem. These models might involve constructing of a vertex-edge graph for problems that involve the relationships between a number of elements, such as Eulerian circuits, minimum spanning trees, and conflict relationships (Hart, 1998, 2008; Hart \& Martin, 2018). Second, students analyse the model and attempt to devise an algorithm to solve the problem. At this point, students may devise algorithms similar to standard algorithms used to solve the problem such as Hierholzer's algorithm (Eulerian circuits), Kruskal's algorithm (minimum spanning tree), and the WelshPowell algorithm (conflict relationships). Finally, the teacher provides the standard algorithm, and the students compare their algorithms with the standard algorithms. Hart hypothesizes that students' competence in algorithmic problem-solving develops through this instructional pattern and provides some anecdotal evidence that students' 
competence in using the graph colouring algorithm is developed through this approach (Hart, 2008; Hart \& Martin, 2018). Indeed, Wetzel, Milicic, and Ludwig (2020) found that three high-achieving 17-year-olds were able to model a shortest path problem and develop an algorithm similar to Dijkstra's algorithm through a comparable instructional pattern.

Existing research, however, appears to provide very little insight into how students make sense of standard algorithms. In particular, more research into how students make sense of the steps in an algorithm, the intermediate results of these steps, or the difficulties that they encounter in using the standard algorithms (cf. Dagdilelis \& Satratzemi, 1998) is needed. The purpose of the present study is to extend the existing literature by exploring an instructional intervention designed to help students make sense of the steps of a standard algorithm. Although the Hungarian algorithm is used as the focus of this study, I envisaged that the design features of the instructional intervention might be applicable to the teaching and learning of other standard algorithms. My study was guided by the following research questions:

Research question 1: How do students make sense of assignment problems and their solutions?

Research question 2: How to students make sense of each step in the Hungarian algorithm?

Research question 3: What difficulties do students encounter as they execute each step in the Hungarian algorithm?

\section{Theoretical Framework}

Given the limited research into the teaching and learning of discrete mathematics, theoretical frameworks for analysing students' learning of discrete mathematics are still emerging. In this study, I developed a hypothetical learning trajectory (HLT) as the basis for investigating how students make sense of each step of the Hungarian algorithm. An HLT is a theoretical construct that refers to a "teacher's prediction as to the path by which learning might proceed” (Simon, 1995, p. 135). According to Simon's model, an HLT has three key components: a learning goal, learning activities, and hypothetical learning processes. In developing the hypothetical learning processes for this HLT, I adopted two existing perspectives on the teaching and learning of discrete mathematics. The first perspective is that discrete mathematics requires students to develop the heuristic process model the general on the particular. Goldin (2010) asserts that:

Often in discrete mathematics, the problems are quite easy in low-number or small-size cases. In elementary combinatorics, students may solve a problem of permutations or combinations through exploratory methods in a lownumber situation, and modeling the general on the particular, frame a conjecture as to the pattern or formula that would apply to a high-number situation. In vertex-edge graph theory, students may solve a problem with a graph having only a few vertices and edges, and likewise modeling the general on the particular, frame a conjecture applicable to a category including much larger graphs. (p. 247) 
Here, Goldin suggests that students might be offered opportunities to develop solutions to simple problems before examining how their solution might be applied to similar problems with more elements. This proposition is similar to Hart's (1998) aforementioned conjecture that students' competence in algorithmic problem-solving is developed through their exploration of a problem and attempts to devise an algorithm to solve that problem. Assignment problems are a form of combinatorics in which solutions to problems involving a small number of elements can be found through exploratory methods. Hence, I anticipated that students would develop a solution to simple assignment problems through exploration prior to the introduction of the Hungarian algorithm.

The second perspective I adopted is that discrete mathematics requires students to engage in sense making and reasoning. In mathematics education, sense making involves "developing understanding of a situation, context, or concept by connecting it with existing knowledge" (National Council of Teachers of Mathematics, 2009, p. 4). Sense making might be viewed as a way of thinking about mathematical procedures and concepts, as well as problem situations and their solutions (Kilpatrick, Swafford, \& Findell, 2001). Mathematical reasoning is a closely related process that refers to the logical conclusions that students make based on the relationships between concepts and situations, given the assumptions underpinning those relationships (Kilpatrick et al., 2001; National Council of Teachers of Mathematics, 2009). Reasoning, therefore, might be considered an instantiation of the way that students make sense of assignment problems and the steps of the Hungarian algorithm.

From these theoretical perspectives, I developed the four-stage HLT shown in Table 1, which is organized according to four categories. The Purpose column describes which aspect of the algorithm is introduced at stage of the HLT. The Problems and Design features columns contain the text of the problems and the specific features of those problems that were incorporated to elicit the sense making and reasoning that I anticipated would emerge at each stage.

\section{Method}

\section{Participants}

Eight Year 12 students (mean age $=17.5$ years) from a large, high-socioeconomic, government-owned secondary school in Australia participated in the teaching experiment. The students were studying General Mathematics and were in their first semester of Year 12. All had passed Year 11 General Mathematics the previous year as well as a summative modelling task in Year 12. The students volunteered to participate in the study, and the students and their parents gave ethical consent to participate.

At the time of the study, the students were studying Unit 3 of the General Mathematics curriculum and had not yet been introduced to graphs and networks. However, in Year 11, the students had experience using matrices to represent the roads connecting towns or communication pathways in a social network. Furthermore, the students had experience learning new mathematical ideas through mathematical modelling and problem-solving. 


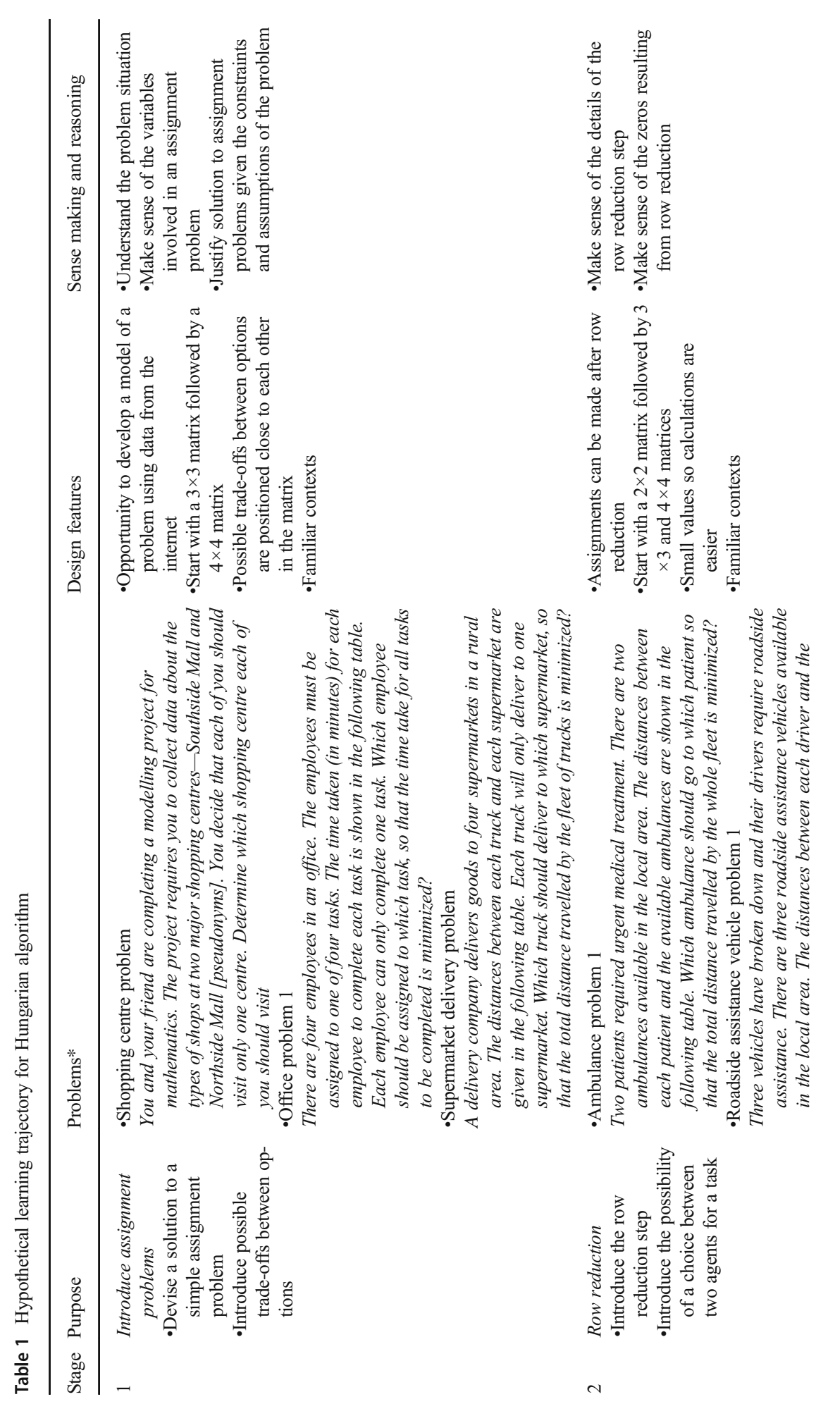




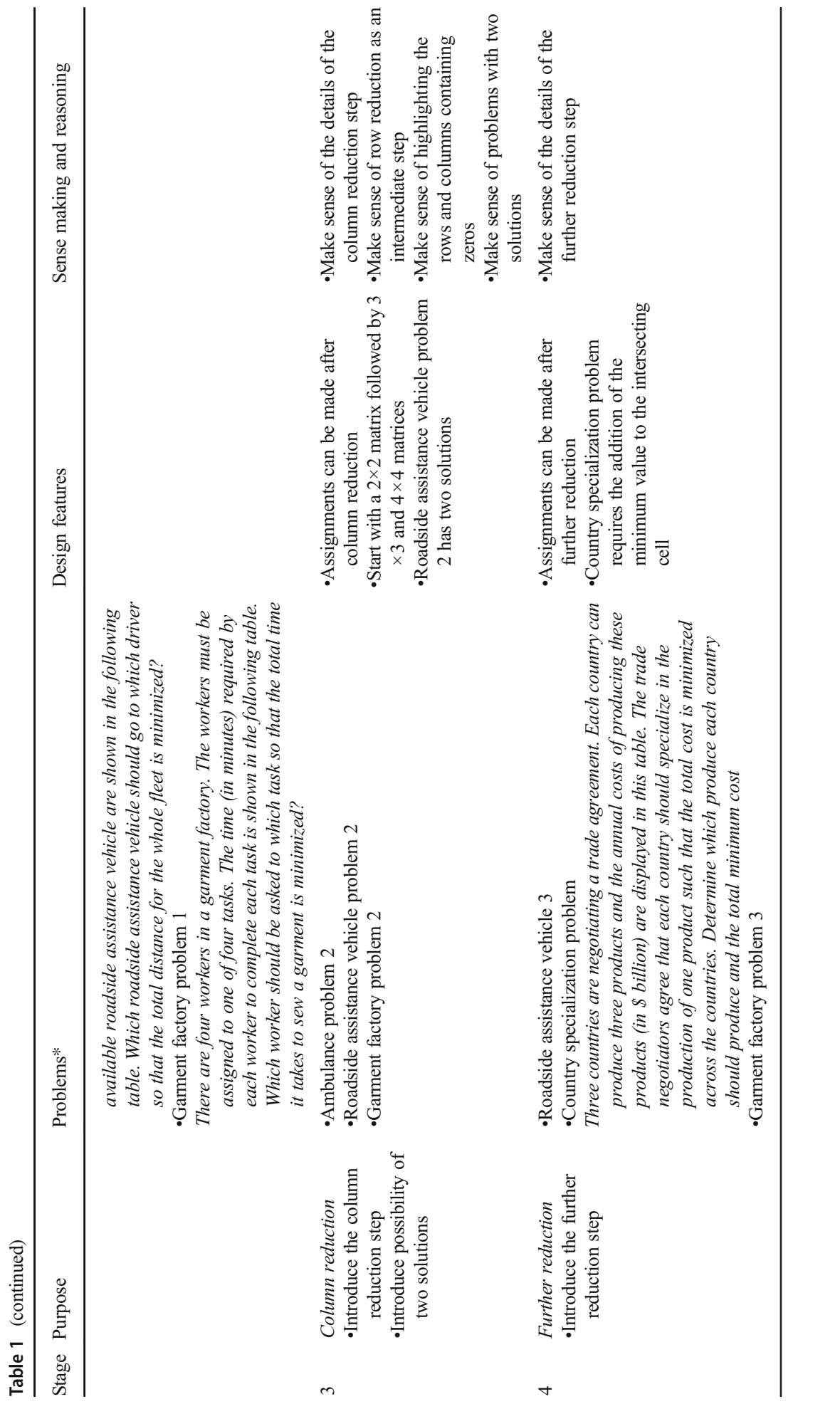




\section{Design}

The present study is part of a larger, design-based research project aimed at improving the teaching and learning of graphs and networks in Year 12 (Cobb, Jackson, \& Dunlap, 2016). In the first cycle of the project, I developed the preliminary instructional intervention and conducted a teaching experiment with one student (Steffe \& Thompson, 2000). I then analysed the results of this pilot teaching experiment and revised the instructional intervention. In the second cycle of the project, I implemented the revised instructional intervention in a series of teaching experiments with the eight students. This article reports the results of this second cycle of the design-based research project. Each cycle of the design-based research project involved three phases, which are described in the remainder of this section (Cobb et al., 2016).

\section{Phase 1: Activity Design}

The design of the instructional intervention began by considering the instructional starting point (Cobb et al., 2016). As stated, the students in this study had limited experience in networks as well as optimum assignment problems. However, they had experience in using matrices to store information and solving problems using this information in matrix form. The students might also have had experience in examining combinations through probability in junior secondary school. Thus, I hypothesized that students might be able to solve simple assignments by inspection using their existing mathematical reasoning at the start of the teaching experiment. The introduction of the procedures in the Hungarian algorithm could then be introduced incrementally from this instructional starting point.

In developing the learning activities for each stage of the HLT, I devised three design heuristics. First, assignment problems for each step of the Hungarian algorithm can be solved after executing that step. That is, problems for the row reduction stage can be solved after the row reduction step is executed, and so on. I hypothesized that this incremental approach would offer students an opportunity to make sense of how each step of the algorithm works to narrow the potential options for an optimum assignment. Second, the problems for each step comprised smaller matrices $(2 \times 2$, $3 \times 3$ ) before problems with larger matrices $(4 \times 4)$. Solutions to problems involving smaller matrices can be found by inspection or by assessing all the possible combinations. Hence, this design feature might offer students an opportunity to examine how the algorithm yields a solution using only a few data points before examining how it works on larger sets of data. The third heuristic was to use contexts that students might readily understand. For instance, the time taken to complete tasks or distances travelled might allow students to interpret the data as they make sense of assignment problem solutions and the steps in the algorithm.

\section{Phase 2: Activity Implementation}

In the second phase, I conducted teaching experiment with each of the students individually (Steffe \& Thompson, 2000). The teaching experiments consisted of four 60-min sessions with each of the eight students ( 24 sessions). These sessions occurred over 5 weeks, which coincided with the COVID-19 pandemic lockdown; hence the students were not attending school. I was the teacher-researcher for each session and conducted the 
sessions over Zoom. During the teaching sessions, I posed the problems by sharing the screen in Zoom. As the student made sense of the problems, I questioned them about their thinking and strategies. The student's working was captured on the shared Zoom screen. The data set included the videos of all sessions captured by Zoom, the saved whiteboard screens from Zoom, and digitized researcher notes taken during the sessions. The audio files from Zoom were transcribed and paired with associated student work.

\section{Phase 3: Retrospective Analysis}

At the end of the teaching experiments, I conducted a retrospective analysis of the data. To complete this retrospective analysis, I first analysed the data and coded students' responses according to three categories, in line with the three research questions: (1) making sense of assignment problems and their solutions, (2) making sense about the steps in the Hungarian algorithm, and (3) difficulties that students encountered in making sense of the Hungarian algorithm. Second, I analysed the transcripts line by line using memos (Corbin and Strauss 2015) and grouped the memos according to qualitatively similar types of sense making and reasoning. Finally, I completed a comparative analysis until no new codes emerged (Corbin and Strauss 2015).

\section{Results}

This section contains the results of the teaching experiment presented in line with the four stages of the HLT. I report the difficulties that students encountered in making sense of the Hungarian algorithm (research question 3) within each of these sections.

\section{Variables}

- Shopping centre locations

- Travel time between each house and shopping centre

\section{Assumptions}

- Times from Google take into consideration traffic

- Going by car

- Leaving from home

\begin{tabular}{|l|c|c|}
\hline & Southside Mall & Northside Mall \\
\hline Me & 29 & 8 \\
\hline Bridgette & 15 \\
\hline
\end{tabular}

Me - Northside Mall (8); Bridgette - Southside Mall (15)

Each should visit the closest shopping centre

Fig. 1 Suzannah's response to shopping centre problem 


\section{Making Sense of Assignment Problems}

I posed the shopping centre problem to offer students an opportunity to model an assignment problem and make sense of the goals and constraints of these problems. Suzannah's response, reproduced in Fig. 1, illustrates how students used their modelling competencies to develop a solution to this problem. First, students identified a relevant variable needed to solve the problem, that is, the distance between each students' home and each shopping centre measured in either kilometres or minutes. Second, students made reasonable assumptions, typically about how they would measure the distances (using the internet), point of departure for each student, and mode of transport. Third, the students collected data from the internet and constructed a matrix of the distances between each student and each shopping centre. Finally, the students solved the problem by searching for the shopping mall that was closest to each students' home. For example, Suzannah found that the Southside Mall was closest to Bridgette and the Northside Mall was closest to her. The results illustrate how the students made sense of the problem and leveraged their mathematical modelling competencies to develop a model of the problem. Furthermore, the students explained that an optimum assignment can be obtained by choosing the closest shopping centre to each student.

After the students solved the shopping centre problem, I posed office problem 1 (Fig. 2). Students used two strategies in their search for the optimum assignments. The first was to search through each column and find the workers with the lowest value for each task. Using this strategy, the students found two options for task B - Blake and Ellis. This finding led the students to compare the alternative tasks for both Blake and Ellis and subsequently assigned Blake to task B and Ellis to task C. The students also noticed that Blake had the shortest time for task D but assigned Hayden to this task because Blake was already assigned to task B. The following explanation, given by Gilda, illustrates this reasoning.

Gilda:

Researcher:

Gilda:

Researcher:

Gilda:

Researcher: Gilda:
We're trying to...okay. Which employee should be assigned to which task? Okay. I know what you're asking now. Can I just tell you the answer? Yes.

So, Task A should be Tatum [Circles Tatum, see Figure 2]. And then...[Pause] Tell me why that.

Because she has the lowest amount of minutes to complete the task. And Task B should be either Blake or Ellis. But I'm going to say Blake because he is high in Task C and D, and Ellis is quicker in Task C. So, Blake for Task B. Task C is Ellis. And Task D is Hayden. Oh wait, that doesn't work. [Pause] Oh wait, yeah it does. [Pause] No it doesn't. [Pause] Oh wait I guess it does. Yeah, yeah it does.

Why does it work?

Because I was like thinking Blake has the shortest time to do it in Task D, but he's already doing something more effective in Task B. And Hayden has the lowest time in Task D compared to everything else.

\begin{tabular}{|c|c|c|c|c|}
\cline { 2 - 5 } \multicolumn{1}{c|}{} & Task A & Task B & Task C & Task D \\
\hline Blake & 11 & 5 & 10 & \\
\hline Hayden & 9 & 12 & 11 & 10 \\
\hline Tatum & 7 & 9 & 8 & 10 \\
\hline Ellis & 9 & 5 & 4 & 7 \\
\hline
\end{tabular}

Fig. 2 Gilda's response to office problem 1 
None of the students calculated the differences between the alternative assignments despite reasoning that their assignments were based on these comparisons.

The second strategy, used by two students, was to search for an optimum assignment by searching for the lowest value in each row. Leonora's justified her strategy as follows:

Leonora:

\begin{abstract}
It's just whatever they have the lowest number on. But, let's say if two of them had like the same lowest number you would pick who had the second lowest number in another place. But obviously it's whoever had the lowest number in each task.
\end{abstract}

It is worth noting that Leonora was also studying economics and initially compared the office assignment problem 1 to specialization in economics, which involves a similar process.

The second problem I posed during this stage was the supermarket delivery problem. The original purpose of this problem was to elicit the students' reasoning about the comparative differences between options when determining an optimum assignment. However, most students acknowledged the potential for such comparisons while solving office problem 1 and subsequently used this reasoning to solve the supermarket delivery problem. Amelia's explanation illustrates this reasoning:

Researcher:

Amelia:

Researcher:

Amelia:
How did you arrive at that decision? [optimum assignment]

Well, I looked at Truck 4 and I was like what is its lowest number? And I was like oh well its lowest number is Supermarket A. And then Truck 3's lowest supermarket was B. And then when I got to Truck 2, its lowest was D, and so was Truck 1, was D. Yes.

And I was like if I do 93 plus 16 it was 120 . That's a lie. It was like 109 or something like that. Is that right? I don't know, I couldn't tell you. And then 80 plus 22 was like 102 , which is 7 less or something like that. So, it was more efficient to do the 80 [Truck 2 to Supermarket D and Truck 1 to Supermarket C].

In making her assignment, Amelia compared the total distances of the alternative combinations, whereas other students compared the difference in distances between supermarkets $\mathrm{C}$ and D for truck $1(6 \mathrm{~km})$ and truck $2(13 \mathrm{~km})$ and made the assignment based on this comparison (see Fig. 3).

\title{
Row Reduction
}

At this stage, students were introduced to the procedure of subtracting the minimum value in each row from each value in that row, as illustrated by Maria's response in Fig. 4. After they executed the step, I asked them how the procedure made sense to

\begin{tabular}{|c|c|c|c|c|}
\cline { 2 - 5 } \multicolumn{1}{c|}{} & Supermarket A & Supermarket B & Supermarket C & Supermarket D \\
\hline Truck 1 & 80 & 97 & 22 & 16 \\
\hline Truck 2 & 94 & 103 & 93 & 109 \\
\hline Truck 3 & 20 & 19 & 34 & 60 \\
\hline Truck 4 & 48 & 88 & 103 & 80 \\
\hline
\end{tabular}

Fig. 3 Amelia's response to supermarket delivery problem 


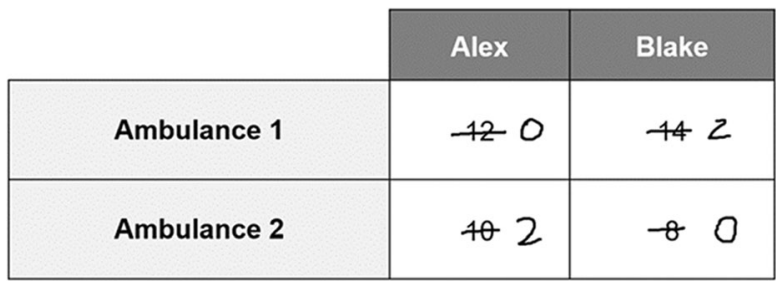

Fig. 4 Maria's response to ambulance problem 1

them. The students' responses revealed that they made sense of the step as a way of determining the differences between the highest and lowest distances for each ambulance. Maria explained her sense making as follows:

Maria:

Oh, I get what this is showing me.

Researcher:

What is it showing you?

Maria:

It's showing me the difference between the, like, whatever one has the lowest amount of difference is the one I should go with.

Researcher: Yep. And what does the 2 represent? [pointing to Ambulance 1 to Blake]

Maria: $2 \mathrm{~km}$.... It's $2 \mathrm{~km}$ different to A.

The students also tended to recognize that the zero values referred to the options that should be selected to determine an optimum assignment.

After the students solved this problem using row reduction, I asked them how they could check if they had made an optimum assignment. The students reasoned that they could check their solution with the alternative combination (Alex, ambulance 2; Blake, ambulance 1), which was $4 \mathrm{~km}$ farther. Three students also equated this $4 \mathrm{~km}$ with the sum of the two residual values.

The students then used the row reduction step to solve roadside assistance problem 1 (Fig. 5). The students applied the same reasoning about the residual values that they developed on the $2 \times 2$ matrix to a $3 \times 3$ matrix.

Garment factory problem 1 (see Leonora's response in Fig. 6) was designed to offer students an opportunity to make sense of an assignment problem in which two workers have the same cost for two different tasks. To find a solution to this problem, students had to make sense of the problem constraint that a worker can be assigned to one task only. Leonora's explanation is indicative of how students reasoned about this problem:

Researcher:

Why did you choose each one? Why did you choose Parker to Overlock?

Leonora:

Because he [Parker] didn't have any other option in the same level of efficiency.

Whereas Quinn had a second option as Sew. So it was pretty clear that, by giving

Parker Overlock, Quinn still had another equally efficient option of Sew. And same with Reece. Reece had an equally efficient option of Press as he did Sew, so may as well give him Press, and Cut Pieces to Sawyer.

\begin{tabular}{|c|c|c|c|}
\cline { 2 - 4 } \multicolumn{1}{c|}{} & Charlie & Dallas & Emmerson \\
\hline Vehicle 1 & $\not 63$ & $\not 50$ & $\not 74$ \\
\hline Vehicle 2 & $\not 20$ & $\$ 2$ & 53 \\
\hline Vehicle 3 & $\not 31$ & 53 & 20 \\
\hline
\end{tabular}

Fig. 5 Anna's response to roadside assistance problem 1 


\begin{tabular}{|c|c|c|c|c|}
\cline { 2 - 5 } \multicolumn{1}{c|}{} & Cut & Overlock & Sew & Press \\
\hline Parker & 727 & 50 & -24 & $\times 2$ \\
\hline Quinn & 704 & 20 & 20 & 22 \\
\hline Reese & 79 & $\times 2$ & -50 & -20 \\
\hline Sawyer & 20 & -21 & -202 & -21 \\
\hline
\end{tabular}

Fig. 6 Leonora's response to garment factory problem 1

Although Leonora tended to use economic language to explain her reasoning, her explanation illustrates how students were able to make sense of the problem constraint and coordinate the zero values to determine the optimum assignment.

\section{Column Reduction}

At the start of the column reduction stage, I asked students to solve ambulance problem 2. The students first completed row reduction, which yielded the matrix depicted in Fig. 7. From this matrix, students observed that Alex was closest to both ambulances, but Blake was closer to ambulance 1 than Alex. Violetta's explanation demonstrates this reasoning:

Violetta:

So again, I did the Row Reduction thingy. And then for both of them, had zero for Alex. But then the leftovers in the Blake column was 2 and 8 . So I chose the one with the lowest leftover as the ambulance for Blake. Because it will be a smaller distance, if you add them together.

Violetta's explanation illustrates how students made sense of the results of row reduction, that is, they searched for the lowest value in the Blake column in order to make the assignment. This rudimentary columnal search illustrates the students' anticipation of the column reduction procedures, which I introduced at this point.

I then posed roadside assistance vehicle problem 2, which required column reduction and contained two possible solutions. All the students carried out the row reduction and then the column reduction, and seven of the eight students recognized that there were two solutions. Leonora explained her reasoning as follows:

\begin{tabular}{|c|c|c|}
\cline { 2 - 3 } \multicolumn{1}{c|}{} & Alex & Blake \\
\hline Ambulance 1 & 10 & 12 \\
\hline Ambulance 2 & 10 & 18 \\
\hline
\end{tabular}

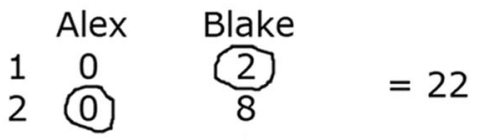

Fig. 7 Violetta's response to ambulance problem 2 
Leonora:

Researcher: Leonora: Researcher: Leonora:
Well, obviously, Dallas as Roadside Assistance Vehicle 2 because that's the most efficient for him. Then Charlie you would want either Roadside Assistance Vehicle 1 or 3. And the same with Emmerson. But you want to choose...well you could kind of swap those. Because, Charlie and Emmerson, if you swapped them over than it's going to be the same. If Charlie gets Roadside Assistance Vehicle 1 instead of Emmerson, he's still going to get 2 kilometers. And if Emmerson did it, it was still going to get the most efficient one.

What did you get as the total?

35 kilometers in total.

What was the other option you said?

It's also 35. It would just be swapping Charlie and Emmerson's ones. But that's also 35 as well. It would just be 15,9 , and 11 .

Leonora's reasoning and response in Fig. 8 illustrate how the students were able to make sense of the results of the algorithm and determine that there were two possible solutions. Maria was the only student who did not immediately recognize that there were two solutions and required a prompt to make sense of this.

Garment factory problem 2 (Fig. 9)required both row reduction and column reduction to find an optimum solution. However, all the students stated their solution after row reduction. For example:

Gilda:

Okay so, looking at my Row Reduction table, Parker should sew, Quinn should cut pieces, and Reece should do Overlock, and Sawyer should Press.

Researcher: Yup, and how did you know?

Gilda: $\quad$ Because Parker and Quinn both had like zero in that [Sew] column. Quinn had the shortest time in cutting pieces compared to everyone else. And that's just how I decided. [See Figure 9]

The students' explanations suggested that they made sense of the residual values from the row reduction step because they argued that the lowest value in the "Cut" column gave the optimum assignment. It is also worth noting that Gilda used the word "zero" to refer to the possible assignments, which indicates that she was beginning to make sense of the zeros as an indicator of options for an optimum assignment.

Three of the students required further clarification of the column reduction step. Specifically, the students questioned if they should execute the reduction procedure on the row reduction matrix or the original matrix. Two students questioned if they should complete column reduction on all the columns or only the columns that contained zeros.

\begin{tabular}{|c|c|c|c|}
\hline & Charlie & Dallas & Emmerson \\
\hline Vehicle 1 & 7520 & 74 & 730 \\
\hline Vehicle 2 & 128 & 2 & $T_{Q}$ \\
\hline Vehicle 3 & $x_{3} \& 0$ & 12 & 710 \\
\hline
\end{tabular}

Fig. 8 Leonora's response to roadside assistance problem 2 


\begin{tabular}{|c|c|c|c|c|}
\hline & Cut & Overlock & Sew & Press \\
\hline Parker & 26 & 31 & 16 & 36 \\
\hline Quinn & 23 & 35 & 21 & 46 \\
\hline Reese & 33 & 21 & 34 & 36 \\
\hline Sawyer & 41 & 31 & 28 & 26 \\
\hline
\end{tabular}

$\begin{array}{cccc}\text { Cut Pieces } & \text { Overlock } & \text { Sew } & \text { Press } \\ 108 & 15 & 0 & 35 \\ 20 & 14 & 0 & 25 \\ 1210 & 0 & 13 & 15 \\ 1513 & 5 & 2 & 0\end{array}$

Fig. 9 Gilda's response to garment factory problem 2

\section{Further Reduction}

At the start of the further reduction stage, I posed roadside assistance vehicle problem 3. After row reduction and column reduction (see Alice's response in Fig. 10), most students anticipated that the optimum assignment would be achieved by sending roadside assistance vehicle 3 to Charlie. For example:

Maria:

Dallas' shortest one was for Roadside Vehicle 1. So, I just kept it there because it's its only zero. Even though Charlie had it too, it's only got an extra 2 kilometers. Whereas Dallas is an extra 7 or 5 kilometers.

Maria's explanation illustrates how six of the students examined the residual values and chose the lowest value to make an assignment. In contrast to this approach, Alice completed a second column reduction on the residual values, as shown in Fig. 10a. This yielded two solutions and I prompted Alice to compare these two solutions, which revealed that the second solution was $3 \mathrm{~km}$ more. This offered the opportunity to explain the first step of further reduction, that is, that the minimum value is subtracted from all uncovered values. Alice subsequently drew a box around these values and carried the further reduction accurately Fig. $10 \mathrm{~b}$.

I posed the country specialization problem to offer students an opportunity to examine the second procedure in the further reduction step, that is, why the minimum value must be added to the values covered by two lines. All the students used row

(a)

\begin{tabular}{|c|c|c|c|}
\cline { 2 - 4 } \multicolumn{1}{c|}{} & Charlie & Dallas & Emmerson \\
\hline Vehicle 1 & 13 & 18 & 12 \\
\hline Vehicle 2 & 12 & 21 & 8 \\
\hline Vehicle 3 & 9 & 17 & 6 \\
\hline
\end{tabular}

Column Reduction

$\begin{array}{cccccccc} & \text { Charlie } & \text { Dallas } & \text { Emmerson } & & \text { Charlie } & \text { Dallas } & \text { Emmerson } \\ 1 & 0 & 0 & 0 & 1 & 0 & 0 & 0 \\ 2 & 3 & 7 & 0 & 2 & 1 & 2 & 0 \\ 3 & 2 & 5 & 0 & 3 & 0 & 0 & 0\end{array}$

Charlie 1, Dallas 3, Emmerson $2=38$

Charlie 3, Dallas 1, Emmerson $2=35$

(b)

\section{Column Reduction}

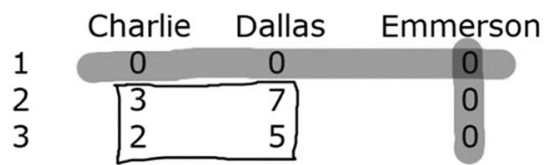

\section{Further Reduction}

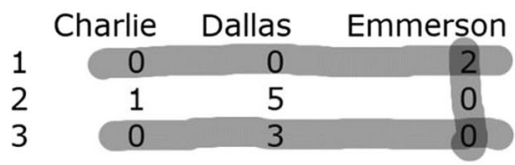

Fig. 10 Alice's responses to roadside assistance vehicle problem 3. (a) Alice's initial response. (b) Alice's response after the procedure was introduced 


\begin{tabular}{|c|c|c|c|}
\hline & Australia & China & Brazil \\
\hline Coffee & 32 & 16 & 24 \\
\hline Wool & 34 & 12 & 18 \\
\hline Toys & 36 & 24 & 26 \\
\hline
\end{tabular}
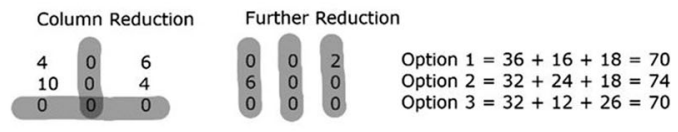

Fig. 11 Amelia's response to country specialization problem

reduction and column reduction, as shown by Amelia's response in Fig. 11 and found that a solution was not possible at this point. The students then subtracted the minimum residual value (4) from the uncovered value and determined that there were three possible solutions. For example, Amelia highlighted three columns and explained the solutions as follows.

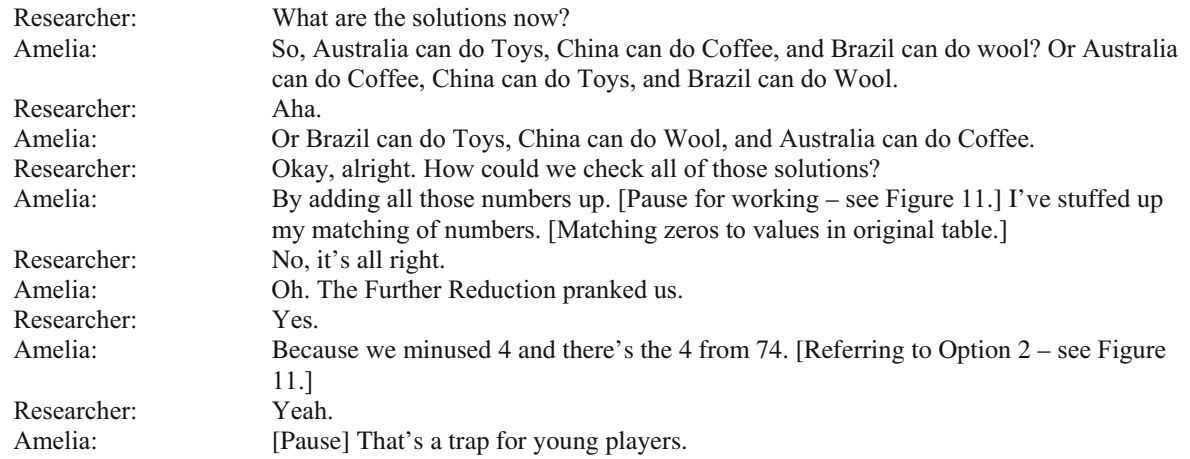

Amelia determined that option 2 would be greater than the other two options by 4 and connected this to the minimum residual value (4) from the first procedure of further reduction. When students reached this point in their reasoning, I connected this residual (4) to the second procedure in the further reduction step.

All the students expressed an opinion about the further reduction step, such as "unusual", "odd", and "creepy". For example, Leonora stated:

That's incredibly random. It just...I feel like they just started running out of ideas and started adding things. I imagine maybe in large scale [problems] you would need this many steps. But usually it's pretty efficiently done in one. You know? People have too much free time.

Despite these opinions, the students were at least able to make sense of the written instructions and appeared to make sense of the experimental demonstration of the further reduction step.

After the students completed these two problems, I asked them to complete garment factory problem 3, which offered them an opportunity to complete the three steps in the Hungarian algorithm. All the students successfully carried out the steps in the algorithm; however, all the students questioned the procedure of highlighting the minimum number of rows and columns. For example, Gilda completed the row and column reduction procedure (see Fig. 12) but highlighted the four rows and explained that each row has a zero. I then prompted her to find a solution, and after searching for a solution, she found that there was not a solution. This provided an opportunity to use an experimental demonstration to help the students make sense of the highlighting procedure. 


\begin{tabular}{|c|c|c|c|c|c|c|c|c|c|c|c|c|}
\hline & Cut & Overlock & Sew & Press & & & & & & & & \\
\hline Parker & 105 & 90 & 135 & 75 & \multicolumn{4}{|c|}{ Row Reduction } & \multicolumn{4}{|c|}{ Column Reduction } \\
\hline Quinn & 100 & 77 & 140 & 75 & 30 & 15 & 60 & 0 & 5 & 13 & 20 & 0 \\
\hline Reese & 120 & 73 & 135 & 70 & $\begin{array}{l}25 \\
50\end{array}$ & $\begin{array}{l}2 \\
3\end{array}$ & $\begin{array}{l}65 \\
65\end{array}$ & $\begin{array}{l}0 \\
0\end{array}$ & $\begin{array}{l}0 \\
25\end{array}$ & $\frac{0}{1}$ & $\begin{array}{l}15 \\
15\end{array}$ & $\frac{0}{0}$ \\
\hline Sawyer & 110 & 85 & 130 & 80 & 30 & 5 & 50 & 0 & 5 & 3 & 0 & 0 \\
\hline
\end{tabular}

Fig. 12 Gilda's row and column reduction for garment factory problem 3

\section{Discussion}

The overarching purpose of the present study was to investigate how students make sense of discrete mathematical problems and the algorithms used to solve those problems. Although assignment problems and the Hungarian algorithm were the focus of the present article, I propose that the results have applicability to other discrete mathematical problems and network algorithms. In reflecting on the results, I consider each of the research questions in turn.

\section{Making Sense of Assignment Problems (Research Question 1)}

The results suggest that the students leveraged their mathematical modelling competence to make sense of assignment problems and the parameters of those problems. Their understanding of assignment problems emerged from a small-scale modelling task at the start of the HLT, and they appeared to carry this understanding forward into the subsequent problems. In particular, the students made sense of the data contained in each of the matrices and the condition that only one agent can be assigned to one task. Furthermore, the students appeared to be aware that they were working towards finding the smallest combination of the agent-task data points. The results suggest that students' competence in mathematizing a problem was a critical factor in making sense of assignment problems and developing rudimentary algorithmic approaches to search for a solution to the problem.

This finding is consistent with Hart's (1998) hypothesis that students' algorithmic problem-solving competence develops through constructing models of problem situations first. However, the results contrast with those of Wetzel et al. (2020), who reported that 17-year-olds experienced difficulty in mathematizing a shortest-path problem because they tended to focus on unnecessary information from a given street map while constructing a vertex-edge graph. The findings of the present study extend the existing literature by illustrating the importance of mathematical modelling competence in making sense of discrete mathematical problems.

\section{Making Sense of the Hungarian Algorithm (Research Question 2)}

The results showed that students developed rudimentary algorithmic approaches to solve the initial assignment problems and assignment problems that contained small matrices $(2 \times 2,3 \times 3)$. Specifically, the students searched through columns and/or rows to find the lowest values that they used to determine the optimum assignment. The students also identified the need to make trade-offs between potential assignments when an agent was more efficient at two tasks than another agent. The students then 
applied their rudimentary search algorithm to solve problems represented by a $4 \times 4$ matrix. This finding is consistent with Goldin's (2010) theoretical perspective that students may solve combinatoric and vertex-edge graph problems that have a small number of elements through exploration and then begin to generalize their algorithmic approaches to solve larger problems. Similarly, researchers hypothesize that students' algorithmic competence is supported by opportunities to devise their own algorithms prior to the introduction of standard algorithms (Gaio \& Di Paola, 2018; Hart, 1998; Hart \& Martin, 2018). In the present study, the problems in the first stages of the HTL were designed to be solved by searching through the rows and assigning agents to tasks based on the lowest values. This design feature appears to have elicited students' rudimentary search algorithms that formed the basis for introducing the Hungarian algorithm in subsequent stages of the HLT.

The results illustrate how students made sense of each step in the Hungarian algorithm and the residual values generated by each step. The students understanding of the Hungarian algorithm had three distinct characteristics. First, students appeared to make sense of the zeros, produced by the reduction procedures, as options for an optimum assignment. Additionally, students were able to examine the zeros and coordinate optimum assignments from these options. Second, most students reasoned that the residual values represented the additional amounts that would be added to an optimum assignment if the option was selected. Finally, the students appeared to make sense of the algorithm, in its entirety, as a method used to find the options available for an optimum assignment.

There are two features of the instructional design that appeared to be crucial in developing students' sense making of the Hungarian algorithm. First, each step of the algorithm was introduced incrementally. This offered opportunities for the students to make sense of both the details of the procedures and the intermediate results produced by those procedures. Second, each step of the algorithm was introduced using smaller matrices. By using smaller matrices, students could compare the results of the algorithm with all possible combinations. These design features were derived from Goldin's (2010) theoretical perspective that students' competence in the heuristic process of modelling the general on the particular develops through solving problems with a small number of elements before making conjectures about how their solution procedures could apply to larger problems. The students in this study lacked the prerequisite knowledge to understand the proof of the Hungarian algorithm and hence the possibility of making conjectures about how their initial search algorithms could be applied to larger problems was limited. However, their initial search algorithms appeared to support the introduction of the steps of the Hungarian algorithm, consistent with Hart's (1998) hypothesized instructional pattern.

\section{Difficulties (Research Question 3)}

The results suggest that students made sense of the Hungarian algorithm as a solution method for assignment problems. However, as predicted by Dagdilelis and Satratzemi (1998), the details of the steps in the Hungarian algorithm are complicated and thus present several challenges for students. First, students questioned if they should perform the column and/or further reduction procedures on the values in the original matrix or on the values resulting from the row and/or column reduction procedure. Second, the students struggled to understand how to highlight the minimum number of rows and/or columns 
containing zeros, and that the number of highlighted rows/columns should equal the number of required assignments. In response to these difficulties, I demonstrated the procedure where necessary and sometimes revised the wording of the steps in the algorithm in collaboration with the students. This finding suggests that teachers might need to take a flexible approach to the wording of the details of an algorithm in response to the way that their students make sense of those details. Dagdilelis and Satratzemi (1998) also suggest that students experience difficulties in making sense of the results of intermediate steps of an algorithm. In the present study, however, students appeared to make sense of the residual values produced by the intermediate steps. The students' apparent understanding of the intermediate results appeared to emerge from their initial experiences with solving problems with a small number of elements. Furthermore, the incremental approach to introducing each step of the algorithm possibly offered students an opportunity to examine the results of the intermediate steps in isolation.

\section{Implications}

Research into the teaching and learning of discrete mathematics is relatively limited, which is surprising given that discrete mathematics topics are of growing importance in high school mathematics curriculums. The results of the present study have three important practical implications for the teaching of network algorithms. First, the incremental approach to introducing an algorithm offered students opportunities to make sense of how each step in the algorithm works. Although the focus in this study was the Hungarian algorithm, I suggest that this incremental approach would also apply to a range of other network algorithms, such as Prim's algorithm, Dijkstra's algorithm, and the Ford-Fulkerson algorithm. Further research might explore how an incremental approach supports students' understanding of these algorithms. Second, the design heuristics that I developed in this study might be used by teachers and researchers in developing problems and activities to enhance students' sense making of network algorithms. Finally, the findings of the present study provide empirical support for the instructional pattern proposed by Hart (1998). Specifically, teachers might offer students opportunities to model and solve discrete mathematical problems before introducing the standard algorithms used to solve those problems.

The generalizability of this study is limited because I used a small sample of students $(n=8)$ from a high socioeconomic school, who were highly engaged and motivated to improve their achievement in mathematics. The instructional intervention was implemented through one-on-one teaching experiments via Zoom during the COVID-19 pandemic. Further research might examine how the instructional approach explored in this study might be adapted to classroom contexts.

Supplementary Information The online version contains supplementary material available at https://doi.org/ 10.1007/s10763-021-10180-3.

\section{Declarations}

Ethics Approval This research was conducted with ethics approval from QUT (QUT Ethics Approval Number 2000000183). 
Informed Consent All participants and their parent/guardian gave informed consent to participate in this study.

Competing Interests The authors declare no competing interests.

\title{
Appendix
}

\author{
Step 1: $\quad$ Row Reduction \\ Determine the minimum value in each row and subtract that value from every \\ value in that row including itself. \\ Cover all the zeroes in the table with the minimum number of horizontal and \\ vertical lines. If the number of lines is equal to the number of allocations \\ required, then go to Step 4.
}

\section{Step 2: $\quad$ Column Reduction}

Determine the minimum value in each column without a zero and subtract that value from every value in the column including itself.

Cover all the zeroes in the table with the minimum number of horizontal and vertical lines. If the number of lines is equal to the number of allocations required, then go to Step 4.

\section{Step 3: $\quad$ Further Reduction}

Determine the minimum value that is not covered by a line and subtract that value from all the uncovered values.

Add the minimum value to all values that are covered by two lines.

Cover all the zeroes in the table with the minimum number of horizontal and vertical lines. If the number of lines is equal to the number of allocations required, then go to Step 4, otherwise repeat this step.

Step 4: Allocate the tasks and calculate the optimum assignment(s).

\section{References}

Australian Curriculum Assessment and Reporting Authority. (2020). General mathematics. Retrieved January 23, 2020 from https://www.australiancurriculum.edu.au/senior-secondary-curriculum/mathematics/

Cobb, P., Jackson, K., \& Dunlap, C. (2016). Design research: An analysis and critique. In L. D. English \& P. A. Kirschner (Eds.), Handbook of international research in mathematics education (3rd ed., pp. 481503). Routledge.

Corbin, J. M., \& Strauss, A. L. (2015). Basics of qualitative research: Techniques and procedures for developing grounded theory (4th ed.). SAGE Publications.

Dagdilelis, V., \& Satratzemi, M. (1998). DIDAGRAPH: Software for teaching graph theory algorithms. ACM SIGCSE Bulletin, 30(3), 64-68.

DeBellis, V., \& Rosenstein, J. (2004). Discrete mathematics in primary and secondary schools in the United States. ZDM, 36(2), 46-55. https://doi.org/10.1007/BF02655758.

Ferrarello, D., \& Mammana, M. F. (2018). Graph theory in primary, middle, and high school. In E. W. Hart \& J. Sandefur (Eds.), Teaching and learning discrete mathematics worldwide: Curriculum and research (pp. 183-200). Springer. https://doi.org/10.1007/978-3-319-70308-4.

Gaio, A., \& Di Paola, B. (2018). Discrete mathematics in lower school grades? Situation and possibilties in Italy. In E. W. Hart \& J. Sandefur (Eds.), Teaching and learning discrete mathematics worldwide: Curriculum and research (pp. 41-51). Springer. https://doi.org/10.1007/978-3-319-70308-4. 
Gibson, J. P. (2012). Teaching graph algorithms to children of all ages. In T. Lapidot (Ed.), Proceedings of the 17th ACM annual conference on Innovation and technology in computer science education (pp. 34-39). Association for Computing Machinery. https://doi.org/10.1145/2325296.2325308.

Goldin, G. A. (2010). Problem solving heuristics, affect, and discrete mathematics: A representational discussion. In B. Sriraman \& L. D. English (Eds.), Theories of mathematics education: Seeking new frontiers (pp. 241-250). Springer. https://doi.org/10.1007/978-3-642-00742-2.

Hart, E. W. (1998). Algorithmic problem solving in discrete mathematics. In L. Morrow (Ed.), Teaching and learning algorithms in school mathematics (pp. 251-267). National Council of Teachers of Mathematics.

Hart, E. W. (2008). Vertex-edge graphs: An essential topic in high school geometry. The Mathematics Teacher, 102(3), 178-185.

Hart, E. W., \& Martin, W. G. (2018). Discrete mathematics is essential mathematics in a 21 st century school curriculum. In E. W. Hart \& J. Sandefur (Eds.), Teaching and learning discrete mathematics worldwide: Curriculum and research (pp. 3-19). Springer. https://doi.org/10.1007/978-3-319-70308-4.

Kilpatrick, J., Swafford, J., \& Findell, B. (2001). Adding it up: Helping children learn mathematics. National Academy Press.

Kuhn, H. W. (1955). The Hungarian method for the assignment problem. Naval Research Logistics Quarterly, 2(1), 83-97.

Makohon, I., Cetin, M., Nguyen, D. T., \& Ng, M. (2016). Hungarian optimum assignment algorithm with Java computer animation. In IEEE SoutheastCon (pp. 1-7). IEEE.

Munkres, J. (1957). Algorithms for the assignment and transportation problems. Journal of the Society for Industrial and Applied Mathematics, 5(1), 32-38.

National Council of Teachers of Mathematics. (2009). Focus in high school mathematics: Reasoning and sense making. National Council of Teachers of Mathematics.

National Research Council. (2013). The mathematical sciences in 2025. The National Academies Press. https://doi.org/10.17226/15269.

Ouvrier-Buffet, C. (2020). Discrete mathematics teaching and learning. In S. Lerman (Ed.), Encyclopedia of mathematics education (pp. 227-233). Springer.

Pérez, A. (2018). A framework for computational thinking dispositions in mathematics education. Journal for Research in Mathematics Education, 49(4), 424-461. https://doi.org/10.5951/jresematheduc.49.4.0424.

Simon, M. A. (1995). Reconstructing mathematics pedagogy from a constructivist perspective. Journal for Research in Mathematics Education, 26(2), 114-145. https://doi.org/10.2307/749205.

Steffe, L. P., \& Thompson, P. W. (2000). Teaching experiment methodology: Underlying principles and essential elements. In R. A. Lesh \& A. E. Kelly (Eds.), Handbook of research design in mathematics and science education (pp. 267-303). L. Erlbaum.

Wetzel, S., Milicic, G., \& Ludwig, M. (2020). Gifted students' use of computational thinking skills approaching a graph problem: A case study. In L. Gómez Chova, A. López Martínez, \& I. Candel Torres (Eds.), Proceedings of EDULEARN20 Conference (pp. 6936-6944). IATED. 